\title{
Plasma sheath properties in a magnetic field parallel to the wall
}

J. Moritz, ${ }^{1, \text { a) }}$ E. Faudot, S. Devaux, and S. Heuraux

Institut Jean Lamour, UMR 7198 CNRS - Université de Lorraine, Bd des Aiguillettes, BP 70239, F-54506 Vandoeuvre-les-Nancy Cedex, France.

(Dated: 25 May 2016)

Particle in cell simulations were carried out with a plasma bounded by two absorbing walls and a magnetic field applied parallel to them. Both the sheath extent and the potential drop in it were derived from simulations for different plasma parameters such as the electron and ion temperature $T_{i}$, particle density and ion mass. Both of them exhibit a power law dependent on the Larmor to plasma ion pulsation ratio $\Omega_{i}$. For increasing values of the magnetic field, the potential drop within the sheath decreases from a few $T_{i} / e$ down to zero, where $e$ stands for the electron charge. The space charge extent increases with $\Omega_{i}$ and saturates to 2.15 ion Larmor radius. A simple model of sheath formation in such a magnetic field configuration is presented. Assuming strongly magnetized electrons, and neglecting collisions and ionizations, a new typical length is evidenced, which depends on the ratio $\Omega_{i}$. The charge separation sheath width is theoretically found to increase from a combination of the electron gyroradius and the ion Debye length for low $\Omega_{i}$ ratios up to several ion gyroradii for strongly magnetized ions. Both the calculated sheath extent and plasma potential show a fair agreement with the numerical simulations.

PACS numbers: 52.40.Kh, 52.65.Rr, 52.40.Fd

Keywords: Plasma sheath, PIC simulations, Magnetic confinement

\footnotetext{
a) jerome.moritz@univ-lorraine.fr
} 


\section{INTRODUCTION}

Sheaths are found in most of studied plasmas for they arise as soon as a plasma encounters a surface. No matter if it is the limiter confining a fusion plasma, the cathode generating a laboratory one or the probe analysing it, the plasma-wall transition is characterized by a spatially positively charged layer, called the Debye sheath ${ }^{1}$, surrounding the surface. Electron velocity being several orders of magnitude larger than its ion counterpart, an electric field builds up in the sheath for slowing down the electrons and speeding up the ions, so that the charge fluxes equal on the wall. The stability of such an electrostatic structure, which extends over several Debye lengths $\left(\lambda_{d}\right)$ in the $x$ direction perpendicular to the wall, requires the ion velocity $v_{i x}$ to exceed the ion sound velocity $\left(c_{s}\right)$ at the sheath edge to satisfy the Bohm criterion ${ }^{2-4}$.

In the presence of a magnetic field $B$ tilted by an angle $\psi$ with respect to the normal to the wall surface, and discarding the two cases where $\psi=0$ (equivalent to the unmagnetized case) and $\psi=\pi / 2$ (investigated in this paper), the plasma-wall transition is split into 3 parts: the Debye Sheath, the magnetic presheath and the collisional presheath. The ions are accelerated along the magnetic lines in the later presheath until their parallel (to the magnetic field) velocity is of the order of $c_{s} \cos \psi$ at the magnetic presheath edge ${ }^{5,6}$. Within the magnetic presheath, also called Chodura sheath, which extents over a few ion Larmor radius, ions are redirected toward the wall in order to satisfy the Bohm criterion at the entrance of the Debye Sheath. The potential drop within the Debye sheath and the magnetic presheath, as well as the role of the magnetic field angle and strength on the different regions have been investigated by several authors since the original work of Chodura ${ }^{7-10}$.

When $B$ is applied parallel to the wall, ions and electrons mobility mainly depends on their Larmor gyroradius ( $R_{L}$ and $r_{L}$ for ions and electrons respectively), leading to a perfectly confined plasma if no other mechanism allows them to drift across the magnetic lines. These mechanisms have been investigated in the literature. For highly collisional plasma, electron mobility is enhanced by collision so that the ions must be accelerated at the vicinity of the wall $^{11,12}$. This leads to a classical negative potential drop within the sheath $(\Delta \phi<0)$. In the collisionless case, Theilhaber and Birdsall showed using 2D particle in cell (PIC) simulations that Kelvin-Helmholtz instability due to velocity shear within the sheath generates an ion flux across the field lines. $\Delta \phi$ is then positive to push the ions back into the plasma and the 
sheath extent $s$ is of $5 R_{L}^{13}$. They also showed that turbulence is suppressed in the general case, i.e. if $B$, still parallel to the wall, is tilted with respect to the axis perpendicular to the simulation plane. This leads to $1 \mathrm{D}$ sheath behaviour with a particle flux null at the wall $^{14}$. In further studies, authors faced the impossibility of solving the Poisson equation from a kinetic theory and used numerical calculations to derive scaling laws. They found that $s$ scales with $R_{L} / \lambda_{d}$ and a $\Delta \phi$ of the order of the ion temperature ${ }^{12,15-17}$, but no simple expression connecting $s$, or $\Delta \phi$, with the plasma parameters such as the ion and electron temperature and mass $\left(T_{i}, T_{e}, m_{i}\right.$ and $m_{e}$ resp.), or with $B$, has ever been derived.

In this paper, we investigate the sheath formation in a parallel to the wall magnetic field, in the case of a perfectly confined plasma with no collision, ionisation nor turbulent fluxes. Such assumptions lead to a particle flux null at the wall once the steady state is reached as explained in ref. ${ }^{14,15}$. The absence of flux at the wall could be seen as very restrictive regarding some experimental studies which have indeed indicated that the flux received by plasma facing components tangential to a flux surface does not vanish due to a possible funneling effect ${ }^{18,19}$, or to turbulent transport or simply to the difficulty to perfectly align the magnetic field with the surface. This is however still an open question as other authors have experimentally shown results at variance with this fact ${ }^{20}$.

Assuming a perfect parallel alignment of the magnetic field with respect to the wall and a perfect confined plasma is justified by the possibility to obtain a scaling of the sheath extent and of the floating wall potential with the magnetic field strength both numerically and analytically.

We show here by using PIC simulations that the potential drop within the sheath and the sheath extent scale with the ion cyclotron to plasma pulsation ratio $\Omega_{i}=\omega_{c i} / \omega_{p i}$. The former decreases from a few $T_{i} / e$ in the weakly magnetized regime of the ions $\left(\Omega_{i}<<1\right)$ to zero for $\Omega_{i}>>1$ where ions are strongly magnetized. The latter increases with $\Omega_{i}$ from a few ion Debye lengths and saturates to $\simeq 2 R_{L}$. In the first part of the paper, the results of the PIC simulations are presented for a series of plasma parameters (density, temperature and ion mass). In the second part, a simple model is derived in order to explain the dependency of the sheath extent and the potential drop within it with respect to $\Omega_{i}$. 


\section{PIC SIMULATIONS}

The simulations have been carried out using a 1D3V PIC code developed in the laboratory by one of the authors. The system studied consists in a plasma bounded by two grounded absorbing walls separated of $15 R_{L}$ with $B$ such as $\omega_{c e}>\omega_{p e}$, where $\omega_{c e}$ and $\omega_{p e}$ are the electron cyclotron and plasma pulsations respectively. A cell size of $\Delta x=0.1 r_{L}$ has been chosen in order to accurately describe both ion and electron motions. Initially, the superparticles are uniformly distributed on the grid $(\sim 100$ per cell) and their velocity set randomly from a Maxwellian distribution. The particles are then moved using the Boris pusher method ${ }^{21}$, with the electric field interpolated at the first order on the particles position. No collision nor particle injection are considered in the numerical scheme. To run the simulations in an acceptable time, we used reduced ion-to-electron mass ratio $m_{i} / m_{e}$ from 200 to $600 . T_{e}$ is kept constant to $2 \mathrm{eV}$ in all calculations, which is a representative value of the electron temperature in plasma discharges ${ }^{22}$.

Fig. 1a and b present the transient particle fluxes at the wall for two different $\Omega_{i}$ ratio. The plasma parameters used in these simulations are $T_{i}=T_{e}=2 \mathrm{eV}, m_{i}=400 m_{e}$ and $n_{0}=10^{16} \mathrm{~m}^{-3}$. Each point in Fig. 1 represents the particle flux integrated over 1000 time iterations. When the ions are weakly magnetized as shown in Fig. 1a $\left(\Omega_{i}=0.08\right)$, the ion flux $\Gamma_{i}$ decreases rapidly during 0.1 ion gyroperiod and completely vanishes after 0.55 ion gyroperiod. The characteristic transient time of $0.1 \times 2 \pi / \omega_{c i}$ is in that case close to $2 \pi / \omega_{p i}$, suggesting the importance of the electric field effects. Note that the electron flux $\Gamma_{e}$ decreases a bit faster than $\Gamma_{i}$ in this regime. When the magnetic field dominates the ion motion in Fig. 1b, for a larger pulsations ratio $\left(\Omega_{i}=1.56\right)$, the ion flux decreases slowly until 0.9 gyroperiod, while the electron flux vanishes after a few time iterations (the electron flux drops to zero after the first point, i.e. in less than 1000 time iterations $=100$ electron gyroperiods). The characteristic transient time is then the ion gyroperiod for large $\Omega_{i}$. In all the following results, the different plasma characteristics such as the ion and electron density $n_{i}$ and $n_{e}$ or the plasma potential vs. $x$ are averaged over $2 \omega_{c i}^{-1}$ once the particle flux has vanished, i.e after one gyroperiod as shown in Fig. 1.

With the aim of emphasizing the role of the electric field in the different regimes of $\Omega_{i}$, the acceleration due to the electric field in the Boris pusher subroutine was forced to zero. Then, the lost particles at the wall, and consequently the densities in the plasma, are determined 

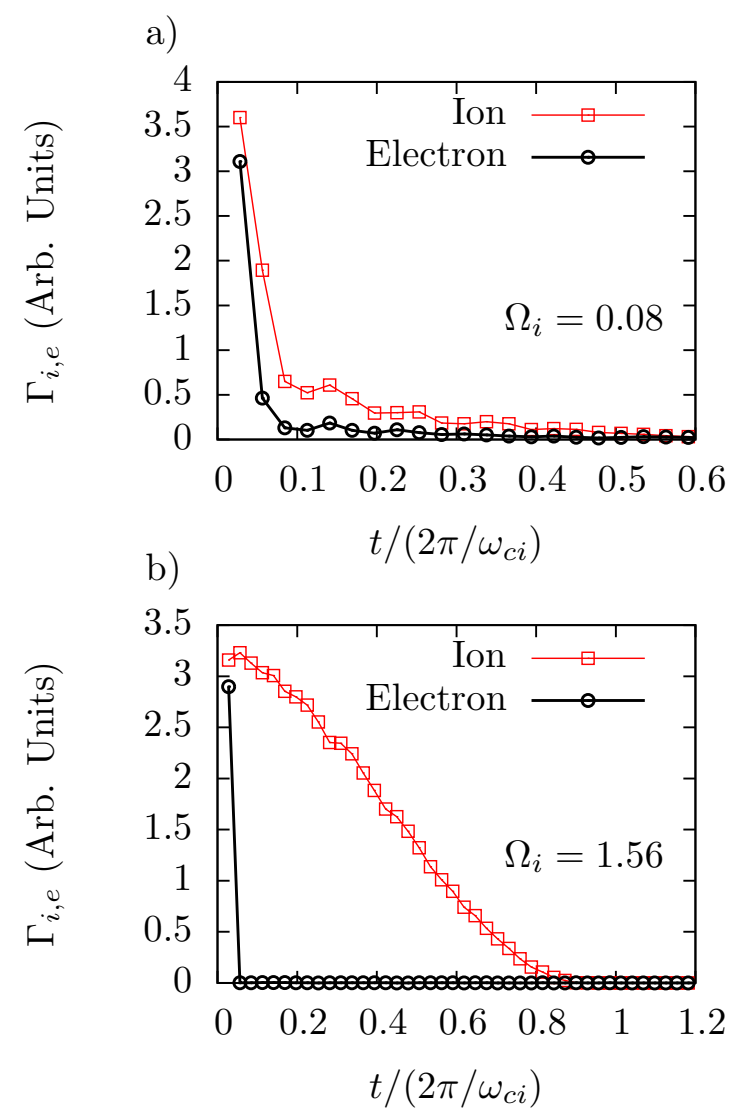

FIG. 1. a) and c) Variation of the ion $\left(\Gamma_{i}\right)$ and electron $\left(\Gamma_{e}\right)$ fluxes with respect to the simulation time for different $\Omega_{i}$ ratio.

by the gyromagnetic motion only. Comparing the densities with $\left(n_{e}\right.$ and $\left.n_{i}\right)$ and without the electric field $\left(n_{e 0}\right.$ and $\left.n_{i 0}\right)$ in Fig. 2, it can be seen that electrons are always strongly magnetized as expected by the magnitude of $B$ in our simulations leading to $\omega_{c e}>\omega_{p e}$. The behaviour of the ions is clearly dependent on the electric field for small $\Omega_{i}$, their density reaching the plasma density at a distance to the wall smaller than $R_{L}$. For large $\Omega_{i}$, the ion density is weakly dependent on the electric field and decreases over several $R_{L}$ in front of the wall.

In both cases, the space charge close to the wall is negative and an electric field builds up in the sheath in order to repel the ions back into the plasma. This is the opposite situation to what is usually observed when the field lines intercept the wall. In Fig. 3 are shown the normalized plasma potential $e \phi / T_{i}$ and the space charge $\rho$ with respect to the distance to the wall $x / R_{L}$ for different $\Omega_{i}$ ratios and for the same plasma parameters as previously. When 
a)
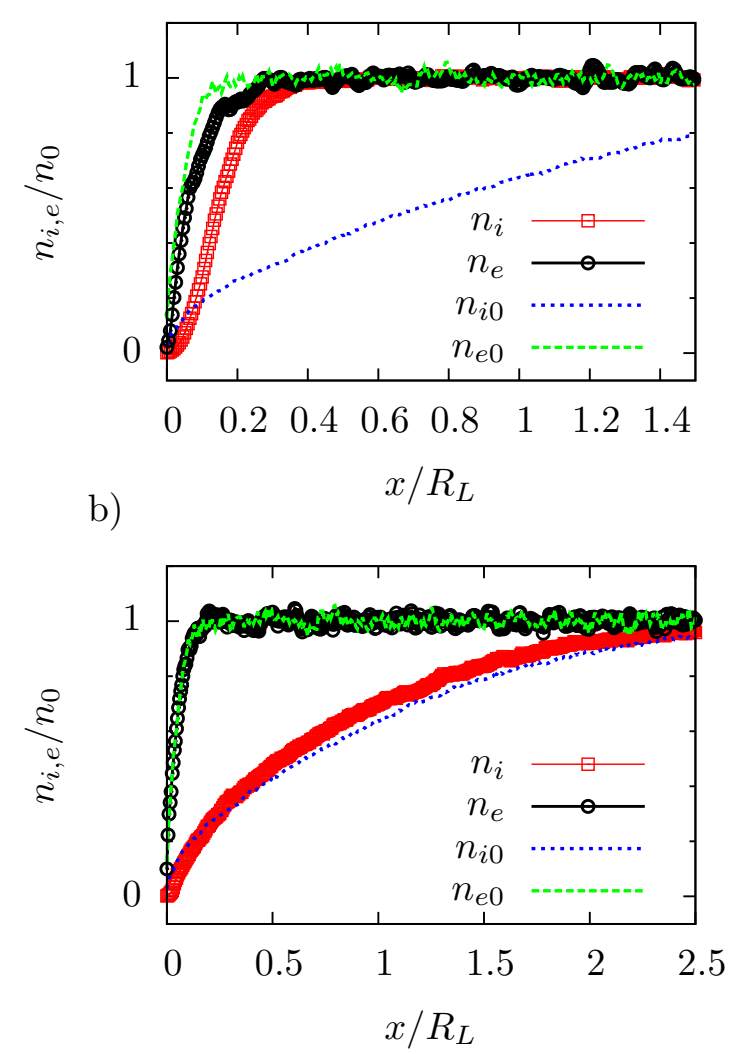

FIG. 2. Ion $\left(n_{i}\right)$ and electron $\left(n_{e}\right)$ density for $\Omega_{i}=0.08$ in a) and $\Omega_{i}=1.56$ in b). The 0 subscript in $n_{i}$ and $n_{e}$ denotes the results obtained when the electric field is artificially taken to be zero in the code. The parameters of the simulation are $T_{i}=2 \mathrm{eV}, m_{i}=400 m_{e}$ and $n_{0}=10^{16} \mathrm{~m}^{-3}$.

$\Omega_{i}$ increases, so does the sheath thickness (the space charge extent), while the potential drop $\Delta \phi$ within it decreases. We analysed the evolution of $\Delta \phi$ and $s$ with respect to $T_{i}, m_{i}$ and $n_{0}$. The sheath thickness was determined using the ion density when $n_{i}=0.9 \times n_{0}$, because of the numerical noise around the saturation and to compare the different results without ambiguity. In Fig.4 are shown the variations of $e \Delta \phi / T_{i}$ and $s / R_{L}$ with respect to $\Omega_{i}$ for the different plasma parameters investigated (the $\Omega_{i}$ sweep is obtained by changing the magnetic field strength keeping $\left.\omega_{c e}>\omega_{p e}\right)$. From Fig. 4a one can see that $e \Delta \phi / T_{i}$ reaches about 3.7 for $\Omega_{i}<<1$ and decreases rapidly for $\Omega_{i}<1$ until it drops out for large $\Omega_{i}$ ratios. The sheath width saturates at $2.15 R_{L}$ for $\Omega_{i}>>1$ and quickly decreases for $\Omega_{i}<1$ (Fig. 4b). It is important to note here that both quantities $(s$ and $\Delta \phi)$ depend on the ion mass, the plasma density and the ion temperature. For instance, if $T_{i}$ or $m_{i}$ are increased, so is the 


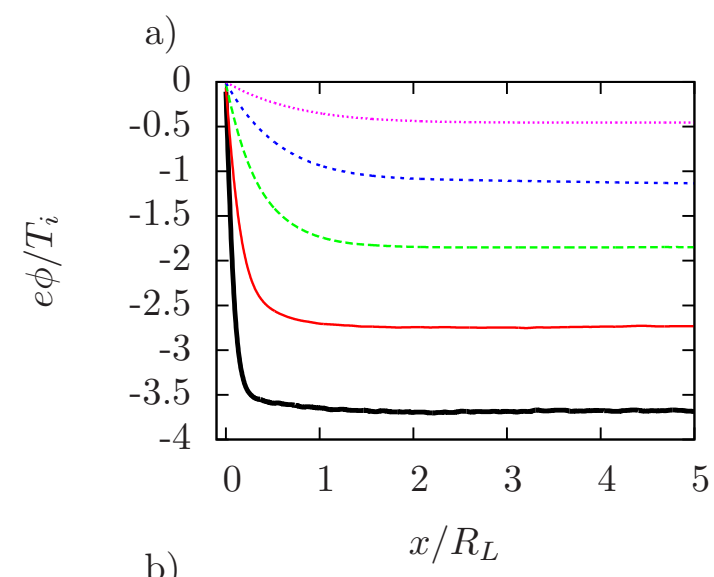

b)

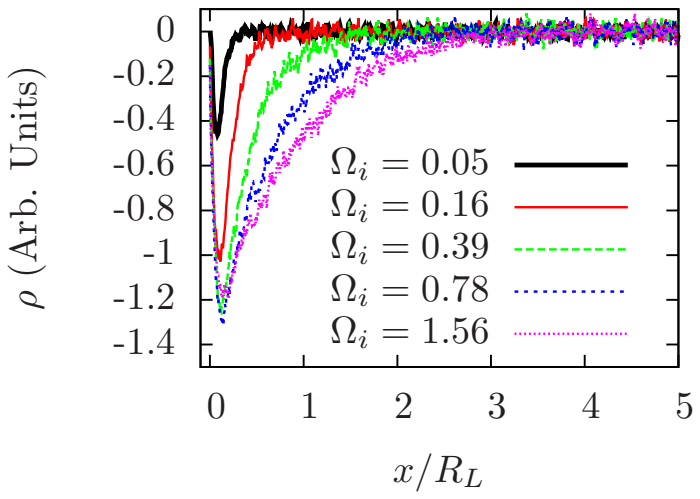

FIG. 3. Variation of the normalized potential $e \phi / T_{i}$ a) and of the space charge $\rho$ in b) with respect to the distance to the wall for different $\Omega_{i}$ ratio.

potential drop within the sheath in absolute units. However, when $s$ and $\Delta \phi$ are expressed in reduced units as in Fig. 4, and plotted with respect to $\Omega_{i}$, every single characteristic is rescaled on an unique master curve. The dependence of both the sheath extent and the potential drop within it is then in $B / \sqrt{m \cdot n_{0}}$ and will be explicitly calculated in the next section. Finally other simulations have been carried out with a larger electron temperature $\left(T_{e}=10 \mathrm{eV}\right.$ and $\left.T_{e}=20 \mathrm{eV}\right)$ and no significant variation to the master curve of Fig. 4 has been evidenced. In this parallel to the wall geometry of the magnetic field, and for such a magnetic field amplitude $\left(\omega_{c e}>\omega_{p e}\right)$, electrons stick to their field line. The dynamic of the ions only drives the sheath characteristics. Within the range of $T_{e}$ that we have investigated, the results of our simulations hold for either plasma discharges or plasma in the scrape-off layer of tokamaks ${ }^{23}$ where the electron temperature is of the order of $25 \mathrm{eV}$. 


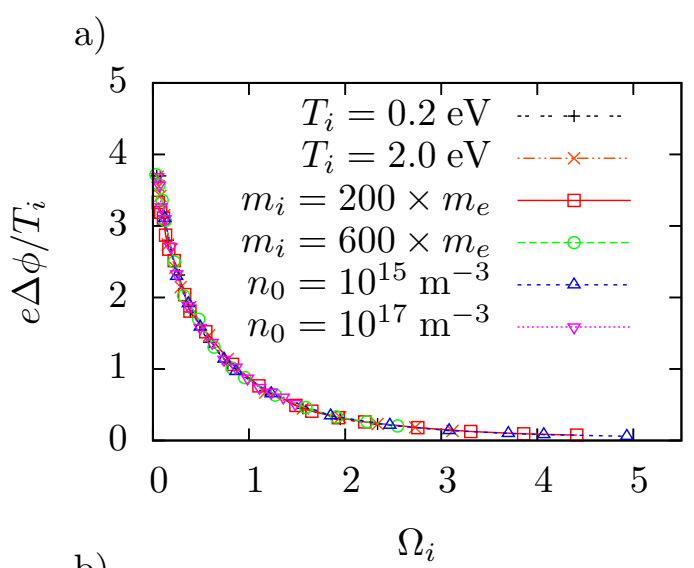

b)

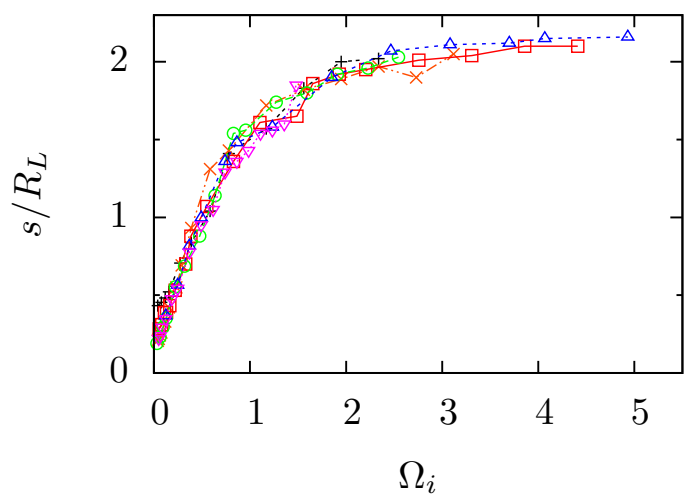

FIG. 4. Normalized potential drop within the sheath a) and sheath thickness b) for different plasma constants. The nominal parameters of the simulation are $T_{i}=2 \mathrm{eV}, m_{i}=400 m_{e}$ and $n_{0}=10^{16} \mathrm{~m}^{-3}$. When one of them is changed in a simulation run, $T_{i}$ for instance, both others are kept constant to the nominal values.

\section{MODEL}

In order to understand the evolution of $\Delta \phi$ and $s$ with respect to $\Omega_{i}$, we propose a very simple model based onto particle motions. We assume a semi-infinite plasma in contact with a conducting and absorbing wall at $x=0$ (see Fig. 5) and we focus on the case of strongly magnetized electrons only, i.e $\omega_{c e}>\omega_{p e}$. Considering electrons at their thermal velocity $v_{t e}$ parallel to the $x$ axis and neglecting the electric field effect on their motion along this direction, after an electronic gyroperiod $2 \pi / \omega_{c e}$, their flux at the wall vanishes. The electronic density $n_{e}(x)$ can then be approximated by a step function: $n_{e}(x)=0$ for $x \leq r_{L}$ and $n_{e}(x)=n_{0}$ otherwise. The ion mobility towards the wall, perpendicular to the field lines, is larger than the electron one $\left(R_{L}>>r_{L}\right)$. As the electronic flux at the wall 
cancels, so does the ion one at the equilibrium, and a positive electric field $E(x)$ arises in the sheath in order to repel ions back into the plasma. Considering a single fluid velocity for the ions $v_{i}$, we assume also that the ion density $n_{i}$ follows a step variation, with $n_{i}(x)=0$ for $x \leq s$ and $n_{i}(x)=n_{0}$ above. Although such a step model (SM) is a crude approximation, it has already been successfully applied to model radio-frequency sheaths ${ }^{24,25}$. The electric field is calculated from the Poisson equation $\partial E / \partial x=\frac{e}{\epsilon_{0}}\left(n_{i}-n_{e}\right)$ for the different regions:

$$
E(x)= \begin{cases}\frac{n_{0} e}{\epsilon_{0}}\left(s-r_{L}\right) & \text { for } x \leq r_{L} \\ \frac{n_{0} e}{\epsilon_{0}}(s-x) & \text { for } r_{L} \leq x \leq s \\ 0 & \text { for } x \geq s\end{cases}
$$

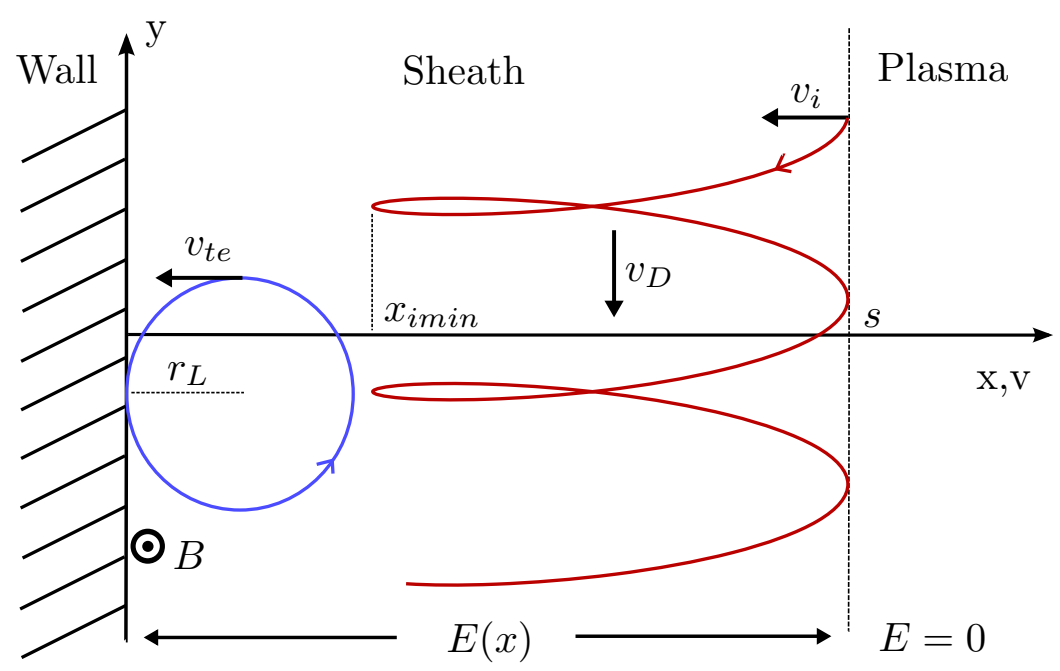

FIG. 5. Trajectories of the plasma particles. $v_{t e}, v_{i}$ and $v_{D}$ are the electron thermal velocity, the ion and the drift velocity respectively. $x_{i m i n}$ is the minimum abscissa reached by the ion originally at the velocity $v_{i}$ under both $B$ and $E(x)$ action.

The aim of the model is to follow the ion motion for the electric field given by Eq. 1 and to self-consistently deduce the minimum $s$ that is necessary to keep the ions into the system $\left(x_{\text {imin }}>0\right.$ in Fig. 5). For ions entering the sheath with a speed $v(x=s)=-v_{i}$ directed toward the wall at $t=0$, it comes from the Newton equations of motion:

$$
\begin{aligned}
& \dot{v_{i x}}=\omega_{c i}\left(\frac{E(x)}{B}+v_{i y}\right) \\
& \dot{v_{i y}}=-\omega_{c i} v_{i x}
\end{aligned}
$$


with $\omega_{c i}$ the ion Larmor pulsation. Differentiating Eq.(2a) with respect to time for $r_{L} \leq x \leq s$ and assuming $\partial E / \partial t=0$ gives $\ddot{v_{i x}}=\omega_{c i} v_{i x}\left(\frac{1}{B} \frac{\partial E}{\partial x}-\omega_{c i}\right)$. With the initial condition $v_{i x}(0)=-v_{i}, v_{i y}(0)=0$ and $E$ from Eq. (1), it comes after integration:

$$
\begin{aligned}
& v_{i x}(t)=-v_{i} \cos \omega_{h i} t \\
& v_{i y}(t)=\frac{v_{i} \omega_{c i}}{\omega_{h i}} \sin \omega_{h i} t
\end{aligned}
$$

with $\omega_{h i}$ the ion hybrid frequency defined as $\omega_{h i}^{2}=\omega_{c i}\left(\omega_{c i}+\frac{n_{0} e}{\epsilon_{0} B}\right)=\omega_{c i}^{2}+\omega_{p i}^{2}$ and $\omega_{p i}$ the ion plasma pulsation.

The time of flight $t_{f}$ of an ion between $x=s$ and $x=r_{L}$ is derived from the integration of Eq.(3a). Replacing $t_{f}$ in Eqs.(3) gives the initial conditions on the ion velocity for its motion through the last region $\left(x \leq r_{L}\right)$ :

$$
\begin{aligned}
& v_{i x 0}=-v_{i} \sqrt{1-\lambda^{2}} \\
& v_{i y 0}=\omega_{c i}\left(s-r_{L}\right)
\end{aligned}
$$

with $\lambda=\omega_{h i}\left(s-r_{L}\right) / v_{i}$. Differentiating the set of equations (2) with respect to time and assuming $v_{i x}$ of the form $\cos \left(\omega_{c i} t+\theta\right)$, and using the initial conditions (4), it comes the ion position $x_{i}\left(t-t_{f}\right)$ for $x \leq r_{L}$ and $t \geq t_{f}$ :

$$
x_{i}\left(t-t_{f}\right)=\frac{v_{\perp}}{\omega_{c i}}\left(\sin \left(\omega_{c i}\left(t-t_{f}\right)+\theta\right)-\sin \theta\right)+r_{L}
$$

with $v_{\perp}^{2}=v_{i x 0}^{2}+\left(v_{D}+v_{i y 0}\right)^{2}, v_{D}=E / B$ is the drift velocity and $\sin \theta=-\frac{v_{D}+v_{i y 0}}{v_{\perp}}$. The minimum drift velocity required to keep the ion inside the plasma is derived from (5). From $x_{i}>0$ and $\sin \left(\omega_{c i} t+\theta\right)=-1$ it comes:

$$
v_{D}>\frac{v_{i x 0}^{2}-\omega_{c i}^{2} r_{L}^{2}}{2 \omega_{c i} r_{L}}-v_{i y 0}
$$

We assume the initial ion velocity at the sheath edge $(x=s, t=0)$ of the form $v_{i}=\sqrt{\frac{\alpha T_{i}}{m_{i}}}$. Replacing $v_{D}=\frac{n_{0} e}{\epsilon_{0}} \frac{s-r_{L}}{B}=\frac{\omega_{p i}^{2}}{\omega_{c i}}\left(s-r_{L}\right), v_{i}, v_{i x 0}$ and $v_{i y 0}$ in Eq.(6) gives for the minimum sheath size:

$$
s^{2}=\frac{r_{L}^{2}+\frac{\alpha}{2} \Omega_{i}^{2} R_{L}^{2}}{1+\Omega_{i}^{2}}=\frac{r_{L}^{2}+\alpha \lambda_{d i}^{2}}{1+\Omega_{i}^{2}}
$$

with $\Omega_{i}=\omega_{c i} / \omega_{p i}$ and $\lambda_{d i}$ the ion Debye length. The potential drop within the sheath $\Delta \phi$ is calculated integrating Eq.1 with the boundary condition $\phi(0)=0$ as:

$$
\frac{e \Delta \phi}{T_{i}}=\frac{\frac{\alpha}{2}-\frac{r_{L}^{2}}{R_{L}^{2}}}{1+\Omega_{i}^{2}}
$$


Neglecting the term $\propto r_{L}$ in equation $8\left(r_{L} / R_{L}<<1\right)$ and assuming $\alpha=2$, it comes $\Delta \phi \simeq T i / e$ for $\Omega_{i} \approx 0$, which is the potential drop required to push back into the plasma a non magnetized ion at the thermal velocity. It is worth noting that this result changes when the ion velocity at the sheath edge increases: the potential drop is then larger to push back faster ions. About $99 \%$ of the ion velocity is contained between 0 and $3 \sqrt{T_{i} / m_{i}}$ in a Maxwellian distribution, so that a potential drop of the order of several Ti/e is expected in the weakly magnetized regime, i.e $\alpha>2$. Moreover $\Delta \phi$ decreases rapidly to zero with $\Omega_{i}$, where the trajectory of strongly magnetized ions $\left(\Omega_{i}>>1\right)$ is slightly affected by electric fields. As the decay of $\Delta \phi$ with $\Omega_{i}$ depends on the density profile chosen, similar calculations have been done with linear density profiles (between the wall and $r_{L}$ for electrons and $s$ for ions) instead of the SM. It leads to a different electric field expression than Eq. (1) on the different regions. The motion of the charged particle is then calculated with the averaged electric field $\bar{E}=\frac{1}{s} \int_{0}^{s} E(x) d x$ in order to derive a full algebraic expression of $s$ and $\Delta \phi$. Actually it only turns $\Omega_{i}^{2}$ into $3 \Omega_{i}^{2}$ in Eq.(7) and (8). Then the potential drop within the sheath decays then more rapidly for the linear model (LM) of particle densities.

In Fig. 6 are shown $e \Delta \phi / T_{i}$ and $s / R_{L}$ with respect to $\Omega_{i}$ for different values of $\alpha$ using Eq.(7) and (8), compared with the curves obtained with the PIC simulations for $n_{0}=10^{15} \mathrm{~m}^{-3}, T_{i}=2 \mathrm{eV}$ and $m_{i}=400 m_{e}$, which extends over the largest range of investigated $\Omega_{i}$. The calculated variation with $\alpha=7.48$, that adjusts the potential drop within the sheath in the weakly magnetized regime of the ions, shows a good tendency with respect to the simulations. The agreement is fairly good using a LM instead of a SM with the same value of $\alpha$, because the decay of $e \Delta \phi / T_{i}$ is accentuated as explained previously. Concerning the sheath size variation, a relative good agreement is found for the investigated $\Omega_{i}$ for both LM and SM. Moreover, according to Eq.7, for $\Omega_{i}<<1, s \simeq \sqrt{\alpha \lambda_{d i}^{2}+r_{L}^{2}}$, so that $s$ ranges between 0.130 and $0.384 R_{L}$ with the different input parameters and $\alpha=7.48$. This is very similar to what is evidenced by the PIC simulations in Fig. 4b. It is also emphasized in Fig. 7 where the evolution of the sheath extent normalized to $R_{L}$ and $\lambda_{d i}$ for one set of the plasma parameters is shown. When $\Omega_{i}<<1$, the sheath width is of about $7.5 \lambda_{d i}$, while it reaches roughly $2 R_{L}$ in the strongly magnetized regime of the ions. Actually, our model holds for $\omega_{c e} \geq \omega_{p e}$, because we neglect the effect of the electric field on the electron motion. It induces a lower bound limit for $B \geq \sqrt{n_{0} m_{e} / \epsilon_{0}}$ and for $\Omega_{i} \geq \sqrt{m_{e} / m_{i}}$. In such a case where $\omega_{c e}=\omega_{p e}, r_{L} \simeq \lambda_{d e}$, the electron Debye length. Assuming $T_{e}=T_{i}$, we have 
a)

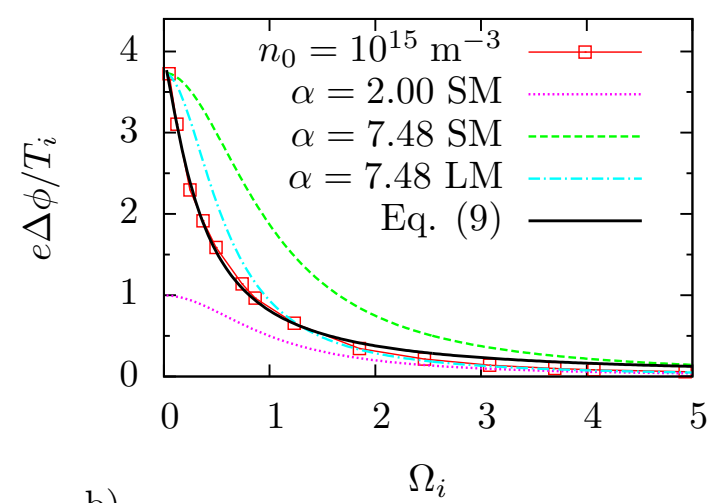

b)

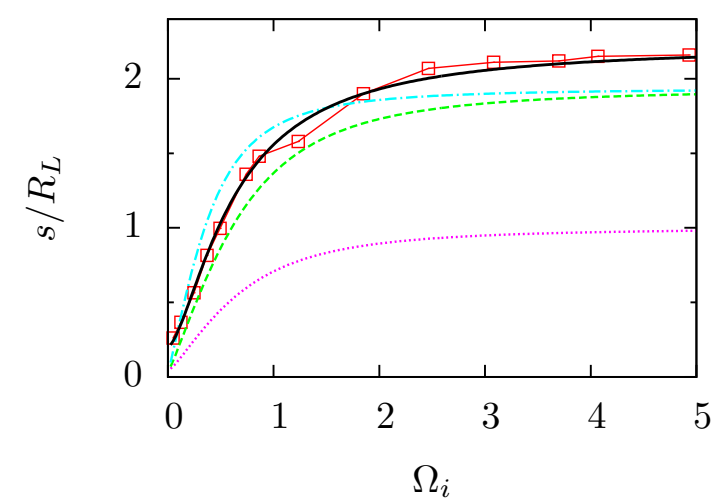

FIG. 6. Variation of both normalized potential drop a) and sheath width b) vs. $\Omega_{i}$. The PIC simulation results are shown for $n_{0}=10^{15} \mathrm{~m}^{-3}$. The calculated curves with various $\alpha$ come from Eq. (7) and (8) respectively. The phenomenological laws (9) are superimposed in both figures.

$\lambda_{d e}=\lambda_{d i}=\lambda_{d}$ and according to Eq. 7 , the sheath extent is of about $\sqrt{\alpha+1} \lambda_{d}$. For weakly magnetized ions, the sheath characteristics do not depend on the magnetic field strength. Then, its extension scales with the Debye length, just like in unmagnetized sheaths (or when the applied field is titled with respect to the wall), but with a negative space charge instead of a positive one.

The results of the PIC simulations and the model evidence 2 regimes for the potential drop and the sheath thickness. When $\Omega_{i}<1$ (as long as $\omega_{c e}>\omega_{p e}$ ), the transient sheath time scales with the plasma frequency instead of the cyclotron frequency. Ions are not considered as magnetized and their mobility is maximum. It induces a maximum potential drop of several $T_{i} / e$ to repel the ions and a sheath length of the order of the ion Debye length. When $\Omega_{i}>1$, both ions and electrons are strongly magnetized in the sheath. The mobility 


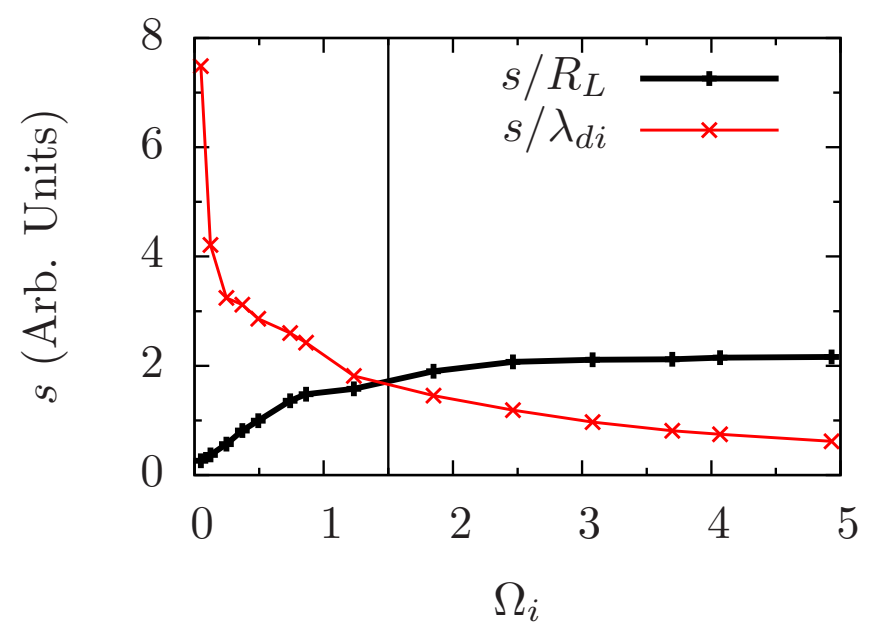

FIG. 7. Variation of the sheath width normalized to the ion thermal Larmor radius $\left(R_{L}\right)$ or to the ion Debye length $\left(\lambda_{d i}\right)$ vs. $\Omega_{i}$. The parameters of the simulation are $T_{i}=2 \mathrm{eV}, m_{i}=400 m_{e}$ and $n_{0}=10^{15} \mathrm{~m}^{-3}$. The vertical line denotes the transition between the weakly and the strongly magnetized regime of the ions.

for ions is reduced but remains higher than the electrons one so that the potential drop stays positive but tends to 0 for strong magnetic fields. The sheath width saturates at 2 times the ion Larmor radius because no other transport mechanism is involved. The discrepancy between simulations and the models (LM and SM) is explained by the approximations made on the electric field and density variation within the sheath. The particle loss calculation at the wall using a Maxwellian velocity distribution and a uniform distribution in space would lead to a gaussian like profile for the ion density. SM and LM are actually zero and first order approximations of the expected exponential density profile using an average particle velocity. Using an average velocity is also a source of error because the particles thermal motion is neglected. In the context of radio-frequency sheaths, the use of a step model for electron density is valid as long as the wall potential is larger than $T_{e} / e^{26}$. Although this assumption is not quantitatively correct in the present work, it is meaningful for understanding the sheath formation and providing a correct order of magnitude of its extension in such a magnetic field configuration. Moreover, using similar power law dependences than previously for $e \Delta \phi / T_{i}$ and $s / R_{L}$, it is possible to find a better agreement with the PIC simulations as shown in Fig. 6. The best agreement compiling all our results was found for a law very similar to the 
one derived in the context of the step model:

$$
\frac{e \Delta \phi}{T i}=\frac{3.89}{1+3.80 \Omega_{i}^{1.29}} \text { and } \frac{s}{R_{L}}=\frac{4.52 \Omega_{i}^{1.53}+0.2}{1+2.03 \Omega_{i}^{1.53}}
$$

with error margins of about $5 \%$ for $e \Delta \phi / T_{i}$ and $10 \%$ for $s / R_{L}$.

\section{CONCLUSION}

We studied the sheath formation in a parallel to the wall configuration of the magnetic field by using PIC simulations and assuming no collision nor flux at the wall except in the transient regime. The numerical results show that the potential drop and the sheath thickness obey to a master curve depending only on the parameter $\Omega_{i}=\omega_{c i} / \omega_{p i}$ and 2 regimes have been identified for the sheath width and potential drop within it. In the weakly magnetized regime of the ions, $\Omega_{i}<1$, the sheath thickness scales as the classical Debye length, while the potential drop decays rapidly from several $T_{i} / e$. In the strongly magnetized regime, the sheath thickness scales with $R_{L}$ and the potential drop slowly decreases to 0 . A new analytic definition of both parameters has been derived from a simple step model of the particle density. From weakly to strongly magnetized conditions, the model yields a sheath width ranging from $\sqrt{\lambda_{d i}^{2}+r_{L}^{2}}$ to $R_{L}$, while the potential drop decreases from $T_{i}$ to 0 , both with the strong dependence on ion cyclotron frequency to ion plasma frequency ratio, as observed in the PIC simulations.

The scaling laws governing the sheath length and potential drop we derived in this paper could be used as boundary conditions in finite element plasma codes in which crossfield sheath are not properly implemented. In tokamak or stellarator devices, most surfaces are almost parallel to the magnetic field and the knowledge of such a sheath is essential to determine the relevant boundary conditions of the codes simulating the scrappe-off layer (for instance JOREK ${ }^{27}$ and SOLPS ${ }^{28}$ ). They can also be useful to floating potential measurements by a Langmuir probe in the special case of the probe collection area being exactly parallel to the magnetic field. Still considering probe, the interpretation of the ion part of the current-tension characteristic requires the knowledge of the sheath size as a function of the applied potential, which could be directly derived from this model. Other PIC simulations have to be run applying such a bias potential. To be applicable this model must obviously be improved by adding collisions or considering a grazing magnetic field angle. 
Finally we may expect that in a quiescent plasma regime with a very low collisionality, such an inversion of the potential drop in the sheath could be observed and would validate the applicability of the present model.

\section{REFERENCES}

${ }^{1}$ L. Tonks and I. Langmuir, Phys. Rev. 34, 876 (1929)

${ }^{2}$ D. Bohm, The Characteristics of Electrical Discharges in Magnetic Fields (A. Guthrie and

R. K. Wakerling, McGraw-Hill, New York, 1949) p. 77

${ }^{3}$ K. U. Riemann, J. Phys. D: Appl. Phys. 24, 493 (1991)

${ }^{4}$ D. D. Tskhakaya, B. Eliasson, P. K. Shukla, and S. Kuhn, Phys. Plasma 11, 3945 (2004)

${ }^{5}$ R. Chodura, Phys. Fluids 25, 1628 (1982)

${ }^{6}$ S. Devaux and G. Manfredi, Phys. Plasma 13, 083504 (2006)

${ }^{7}$ E. Ahedo, Phys. Plasma 4, 4419 (1997)

${ }^{8}$ P. Stangeby, Nuclear Fusion 52, 083012 (2012)

${ }^{9}$ K. U. Riemann, Phys. Plasma 1, 552 (1994)

${ }^{10}$ D. D. Tskhakaya and L. Kos, Phys. Plasma 21, 102115 (2014)

${ }^{11}$ D. D. Tskhakaya, P. K. Shukla, B. Eliasson, and S. Kuhn, Phys. Plasma 12, 103503 (2005)

${ }^{12}$ D. L. Holland, B. D. Fried, and G. J. Morales, Phys. Fluids B 5, 1723 (1993)

${ }^{13}$ K. Theilhaber and C. K. Birdsall, Phys. Rev. Lett. 62, 772 (1989)

${ }^{14}$ K. Theilhaber and C. K. Birdsall, Phys. Fluids B 1, 2244 (1989)

${ }^{15}$ N. S. Krasheninnikova, X. Tang, and V. S. Roytershteyn, Phys. Plasma 17, 057103 (2010)

${ }^{16}$ N. S. Krasheninnikova and X. Tang, Phys. Plasma 17, 063508 (2010)

${ }^{17}$ M. J. Gerver, S. E. Parker, and K. Theilhaber, Phys. Fluids B 2, 1069 (1990)

${ }^{18}$ P. Stangeby, C. Pitcher, and J. Elder, Nuclear Fusion 32, 2079 (1992)

${ }^{19}$ G. Arnoux, T. Farley, C. Silva, S. Devaux, M. Firdaouss, D. Frigione, R. Goldston, J. Gunn, J. Horacek, S.Jachmich, P. Lomas, S. Marsen, G. Matthews, R. Pitts, M. Stamp,

P. Stangeby, and J.-E. Contributors, Nuclear Fusion 53, 073016 (2013)

${ }^{20}$ G. Matthews, D. Hill, and M. A. Mahdavi, Nuclear Fusion 31, 1383 (1991)

${ }^{21}$ J. Boris, Proceedings of the Fourth Conference on Numerical Simulation of Plasmas (Naval Research Laboratory, Washington DC) 1, 3 (1970)

${ }^{22} \mathrm{M}$. Lieberman and A. Lichtenberg, Principles of plasma discharges and material processing 
(Wiley-Interscience, 2005) p. 8

${ }^{23}$ P. C. Stangeby, The Plasma Boundary of Magnetic Fusion Devices (Institute of physics Publishing, London, 2000) p. 21

${ }^{24}$ M. A. Lieberman, IEEE Trans. Plasma Sci. 16, 638 (1988)

${ }^{25}$ V. A. Godyak and N. Sternberg, Phys. Rev. A 42, 2299 (1990)

${ }^{26}$ R. Brinkmann, J. Phys. D: Appl. Phys. 42, 194009 (2009)

${ }^{27}$ G. Huysmans and O. Czarny, Nuclear Fusion 47, 659 (2007)

${ }^{28}$ S. Wiesen, D. Reiter, V. Kotov, M. Baelmans, W. Dekeyser, A. Kukushkin, S. Lisgo, R. Pitts, V. Rozhansky, G. Saibene, I. Veselova, and S. Voskoboynikov, J. Nuc. Mat. 463, $480(2015)$ 